\title{
Don't Anger the Host: New Etiquette in Standard Cancer Assessment?
}

\author{
Kei Suzuki, MD, and Virginia R. Litle, MD \\ Division of Thoracic Surgery, Department of Surgery, Boston University School of Medicine, Boston, MA
}

Immune responses within the tumor microenvironment are increasingly implicated as markers of malignant progression and aggression. Interactions between tumor, immune cells, and cytokines can shift the tumor's microenvironment to welcoming or hostile. ${ }^{1}$ This host-tumor interaction may be reflected in the peripheral blood, and investigation of the host inflammatory response in the peripheral blood of cancer patients may potentially provide a simple tool for clinical decision-making.

In their study, McSorley et al. sought to investigate the prognostic value of the host inflammatory response in patients with locoregional esophagogastric cancer. ${ }^{2}$ They measure the host inflammatory response by a combination of simple preoperative blood work, neutrophil-to-lymphocyte ratio (NLR), and modified Glasgow Prognostic Score (mGPS), demonstrating that NLR and mGPS are associated with response to neoadjuvant chemotherapy and, more importantly, that they are independently associated with overall survival.

While TNM (tumor, nodal, metastasis) staging is the classification system for esophageal cancer, it is primarily derived from gross tumor characteristics including tumor depth, number of involved nodes, and extent of disease. Robust biologic factors are necessary to predict treatment response and overall prognosis. Beyond the TNM staging system, assessment of the tumor-immune microenvironment and the host immune/inflammatory response offers an intriguing and promising tool, particularly with recent advances in immunomodulatory therapies. In other solid

(C) Society of Surgical Oncology 2020

First Received: 25 August 2020

Accepted: 13 October 2020;

Published Online: 27 October 2020

V. R. Litle, MD

e-mail: Virginia.litle@bmc.org malignancies, detailed assessment of the tumor-immune microenvironment in the form of type, location, and density of tumor-infiltrating immune cells has shown prognostic value, often surpassing the prognostic power of the TNM staging system. ${ }^{3}$ In colorectal cancer, the value of such analysis has led some groups to introduce the idea of an "immunoscore" and a TNM-I staging system to integrate the host immune/inflammatory response. ${ }^{4}$

Assessment of tumor-host interaction can also be performed in the peripheral blood. While the tumor-associated immune response within the tumor plays an important role in tumor progression, this response often extends beyond the tumor microenvironment into the peripheral blood. Measuring immune markers in peripheral blood provides easy access to the systemic inflammatory state in response to cancer, as well as providing prognostic and predictive information without the need to access the tumor microenvironment. NLR is known to be an indicator for systemic inflammatory response, and under pathologic stress there may be an increase in the number of neutrophils as well as a simultaneous decrease in lymphocytes, causing an increase in measured NLR. A neutrophilic response can have a negative immunomodulatory effect on cancer cells by suppressing cytotoxic T-cells, and likewise, a decreased lymphocyte response can aid in tumor invasion and progression due to impaired cell-mediated immunity. ${ }^{5}$ The prognostic value of NLR has been shown in esophageal cancers ${ }^{6}$ and other gastrointestinal malignancies, including colorectal and gastric cancers. ${ }^{7,8}$ In esophageal squamous cell carcinoma, NLR has been shown to be prognostic in those treated with definitive chemoradiotherapy. ${ }^{9}$ In addition, for patients receiving trimodality therapy, the change in NLR before and after chemoradiotherapy is associated with treatment response and recurrence. ${ }^{10}$ Another simple peripheral blood inflammatory marker is platelets, as the cancer-associated inflammatory response contributes to 
thrombocytosis. Platelet-to-lymphocyte ratio (PLR) has been investigated as a potential prognostic marker in many solid malignancies, including esophageal cancers. ${ }^{11}$

The mGPS was originally described by McMillan, and is obtained from C-reactive protein (CRP) and albumin. ${ }^{12}$ It is thought to be a marker of chronic inflammatory response, and its prognostic value has been investigated in multiple studies including over 30,000 patients with various cancers. In addition, a combined evaluation of NLR and mGPS has been studied in other solid malignancies. ${ }^{13}$

McSorley et al. demonstrate the predictive and prognostic value of a simple stratification tool combining mGPS and NLR for esophagogastric cancer (93\% adenocarcinoma). The mGPS derives from the following (points ranging from 0-2): CRP $\leq 10 \mathrm{mg} / \mathrm{L}=0 ; \quad C R P>10 \mathrm{mg} / \mathrm{L}$ and albumin $\geq 35 \mathrm{mg} /$ $\mathrm{L}=1 ; \quad \mathrm{CRP}>10 \mathrm{mg} / \mathrm{L}$ and albumin $<35 \mathrm{~g} / \mathrm{L}=2$. One downside of these markers is that NLR and CRP values could vary due to other ongoing acute infectious/inflammatory events in addition to the host response to cancer. On the other hand, the beauty of the system is that it is derived from laboratory values that are routinely collected in everyday practice. These peripheral blood inflammatory markers are low cost, easy to collect, and readily translatable. This is a clear advantage over the more intricate analysis of the tumor-immune microenvironment. The authors demonstrated that patients with low NLR $(\leq 3)$ fared better than those with high NLR, regardless of the mGPS. Among those with high NLR, the authors demonstrated poorer survival with increasing mGPS. Based on these findings, the authors devised a simple scoring system, which groups the 239 patients as NLR $\leq 3$ (least inflamed; best outcome), $\mathrm{NLR}>3+\operatorname{mGPS}=0$, and NLR $>3+$ mGPS $>0$ (most inflamed; worst outcome). The most inflamed group had a significantly higher rate of progression on chemotherapy, as well as a lower proportion of eventual complete resection compared with the others. Similar association was seen between the scoring system and survival: more inflammation correlated with worse outcome. The scoring system remained independently prognostic even on their multivariate analysis including clinical stage and resection status.

Esophageal cancer with its poor survival rate needs more guests at the table to liven the management conversation and subsequent outcomes. How can we best differentiate chemotherapy responders from nonresponders to better direct clinical management? Circulating tumor studies are showing promise yet not with high throughput. ${ }^{14}$ McSorley et al. should be commended for introducing a scoring system based on a blood draw, as it allows us to learn to look beyond the misbehaving tumor and to engage with the host and their guests. In the age of immunomodulatory therapies, the host immune/inflammatory response is a continually important aspect of treating the patient. Keep it simple! We may be a test-tube away from improving esophagogastric cancer outcomes.
DISCLOSURES The author has no potential conflicts of interest.

\section{REFERENCES}

1. Suzuki K, Kachala SS, Kadota K, et al. Prognostic immune markers in non-small cell lung cancer. Clin Cancer Res. 2011;17(16):5247-56.

2. McSorley ST, Lau HYN, McIntosh D, et al. Staging the tumor and staging the host: Pretreatment combined neutrophil lymphocyte ratio and modified Glasgow Prognostic Score is associated with overall survival in patients with esophagogastric cancers undergoing treatment with curative intent. Ann Surg Oncol. 2020. https://doi.org/10.1245/s10434-020-09074-5.

3. Pages F, Mlecnik B, Marliot F, et al. International validation of the consensus Immunoscore for the classification of colon cancer: a prognostic and accuracy study. Lancet. 2018;391(10135): 2128-39.

4. Galon J, Mlecnik B, Bindea G, et al. Towards the introduction of the 'Immunoscore' in the classification of malignant tumours. $J$ Pathol. 2014;232(2):199-209.

5. Sulibhavi A, Asokan S, Miller MI, et al. Peripheral blood lymphocytes and platelets are prognostic in surgical pT1 non-small cell lung cancer. Ann Thorac Surg. 2020;109(2):337-42.

6. Pirozzolo G, Gisbertz SS, Castoro C, van Berge Henegouwen MI, Scarpa M. Neutrophil-to-lymphocyte ratio as prognostic marker in esophageal cancer: a systematic review and meta-analysis. $J$ Thorac Dis. 2019;11(7):3136-45.

7. Zhang J, Zhang H-Y, Li J, Shao X-Y, Zhang C-X. The elevated NLR, PLR and PLT may predict the prognosis of patients with colorectal cancer: a systematic review and meta-analysis. Oncotarget. 2017;8(40):68837-46.

8. Jin H, Sun J, Zhu K, et al. The prognostic value of neutrophillymphocyte ratio is superior to derived neutrophil-lymphocyte ratio in advanced gastric cancer treated with preoperative chemotherapy and sequential R0 resection: a 5-year follow-up. OncoTargets Therapy. 2017;10:2655-64.

9. Zhou XL, Li YQ, Zhu WG, et al. Neutrophil-to-lymphocyte ratio as a prognostic biomarker for patients with locally advanced esophageal squamous cell carcinoma treated with definitive chemoradiotherapy. Sci Rep. 2017;7:42581.

10. Barbetta A, Nobel TB, Sihag S, et al. Neutrophil to Lymphocyte ratio as predictor of treatment response in esophageal squamous cell cancer. Ann Thorac Surg. 2018;106(3):864-71.

11. Yodying H, Matsuda A, Miyashita M, et al. Prognostic significance of neutrophil-to-lymphocyte ratio and platelet-tolymphocyte ratio in oncologic outcomes of esophageal cancer: A systematic review and meta-analysis. Ann Surg Oncol. 2016;23(2):646-54.

12. McMillan DC. The systemic inflammation-based Glasgow Prognostic Score: a decade of experience in patients with cancer. Cancer Treat Rev. 2013;39(5):534-40.

13. Dolan RD, Lim J, McSorley ST, Horgan PG, McMillan DC. The role of the systemic inflammatory response in predicting outcomes in patients with operable cancer: Systematic review and meta-analysis. Sci Rep. 2017;7(1):16717.

14. Egyud M, Tejani M, Pennathur A, et al. Detection of circulating tumor DNA in plasma: a potential biomarker for esophageal adenocarcinoma. Ann Thorac Surg. 2019;108(2):343-9.

Publisher's Note Springer Nature remains neutral with regard to jurisdictional claims in published maps and institutional affiliations. 